\title{
Assessment of pain with mechanical nociceptive stimuli by the change of arterial wall impedance
}

\author{
Abdugheni KUTLUK ${ }^{1}$, Harutoyo HIRANO ${ }^{2}$, Ryuji NAKAMURA ${ }^{3}$, Noboru SAEKI ${ }^{3}$, Masao YOSHIZUMI ${ }^{3}$, \\ Masashi KAWAMOTO ${ }^{3}$, Murat HAMIT ${ }^{1}$, and Toshio TSUJI ${ }^{2}$ \\ ${ }^{1}$ College of Medical Engineering Technology, Xinjiang Medical University, Urumqi, China 830-011 \\ ${ }^{2}$ Graduate School of Engineering, Hiroshima University, Higashi-Hiroshima, Japan 739-8527 \\ ${ }^{3}$ Graduate School of Biomedical Sciences, Hiroshima University, Hiroshima, Japan 734-8551
}

\begin{abstract}
The understanding and treatment of pain is one of the oldest challenges in the field of clinical medicine. In this study, as a first step toward adequate pain assessment, we propose a method to evaluate the reactions of the automatic nervous system in response to painful stimuli by observing arterial wall impedance. Under the proposed method, the mechanical impedance (stiffness) of the arterial wall is calculated from blood pressure and photoplethysmogram measurements on a beat-tobeat basis. In the experiments, we tested eight male subjects (aged $22-23)$ by applying external forces $(1-3[N])$ to the central parts of their palms as painful stimuli, and evaluated changes in levels of arterial wall stiffness during stimulation. The results indicated that stiffness during stimulation showed a significant increase $(p=0.007, p=0.014$ and $p=0.018$ for the stimulus changes from 0 to 1 [N], 1 to $2[N]$ and 2 to 3 [N] for all subjects). We also compared the coefficients of variation in the measured stiffness and visual analog scale (VAS) values during stimulation, and found that the mean coefficients of variation for stiffness $(0.37,0.27$ and 0.26 for the stimuli of 1,2 and 3 [N] for all subjects, respectively) were smaller than the ones of the VAS values $(0.67,0.51$ and 0.50 , respectively). From these results, it was confirmed that changes in the level of measured stiffness can be used to quantify the level of pain felt by a patient.
\end{abstract}

Keywords-pain assessment; arterial wall impedance; photoplethysmogram; visual analog scale

\section{INTRODUCTION}

Many treatments in the field of clinical medicine can be painful, but enabling medical doctors to evaluate patients' pain levels quantitatively and objectively would enable them to reduce this pain burden. By way of example, a doctor could reduce excessive stimulation that burdens a rehabilitation patient or regulate the depth of anesthesia administered [1]. However, it is very difficult to evaluate patients' levels of pain quantitatively and objectively using current technology. A conventional method has been to evaluate pain levels on the Visual Analog Scale (VAS) [2]-[4]. This presents a range from "No pain" to "Worst pain ever" on paper, and the patient marks a point on it to indicate the level of pain felt. VAS is one method to evaluate pain [3], while other techniques also take a relative approach, including the numerical rating scale (NRS) and the face rating scale (FRS) [5]. However, these evaluations are far from objective because the standard of the pain expressed depends on experience and judgment, so it can be said that VAS evaluates patients' pain levels subjectively [6].

Against this background, some previous studies have tried to evaluate pain objectively by measuring and assessing physiological reactions such as autonomic activity and brain nerve activity in response to painful stimuli [7]-[8]. As examples, evaluation of brain nerve activity has been performed using magnetoencephalograpy (MEG) [8]. However, measurement in the case of MEG is limited because of the large-scale equipment required and the strong magnetic fields involved. It is also thought that the evaluation method involving autonomic nerve activity in previous studies exerts a strong emotional influence connected to factors such as the degree or fear of the related burden [9]. On the other hand, a pain evaluation device called Pain Vision (produced/sold by Osachi Co.) has been developed that uses electric stimulus signals to measure pain sensation [10]. This device measures the minimum perceived current and the pain-equivalent current, and quantifies patients' pain levels using an original operation expression [10]. However, this is far from being an objective evaluation because the final decision of the painequivalent current is decided by the patient.

Previously, our research group proposed a noninvasive measurement method of indices for arterial dynamic factors such as stiffness and viscosity to be connected with the autonomous nerve system, and succeeded in diagnosis of success or failure in a hyperhidrosis operation [11]. However, the relationship between blood-vessel viscoelasticity and pain stimulation has not yet been fully elucidated. If it is possible to clarify viscoelasticity changes in response to pain stimulation, then pain can be estimated using noninvasive measurement of viscoelasticity changes in real time. This approach can also be applied to reduce the pain burden of patients in treatments such as dental work and rehabilitation therapy. In this paper, as a first step, we define the stiffness and viscosity of the arterial wall as an arterial wall viscoelasticity index, and report on changes in this index in response to mechanical pain stimuli. 


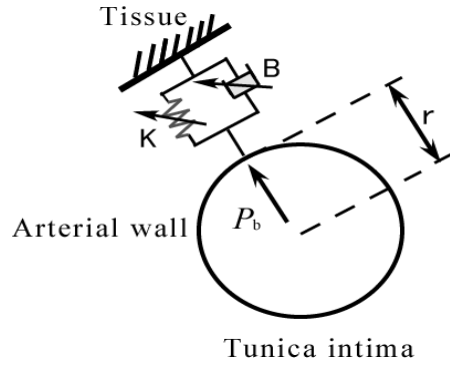

Figure 1. Schematic model of arterial wall impedance

\section{METHOD}

\section{A. Arterial Wall Impedance Model}

In the estimation unit, arterial wall viscoelasticity is estimated in relation to each heartbeat. Figure 1 illustrates the proposed impedance model of the arterial wall; only the characteristics of the wall in an arbitrary radius direction are represented. The impedance characteristic can be described using the arterial blood pressure and plethysmogram as the stress and displacement of the arterial wall as follows [12]:

$$
d P_{b}(t)=\widetilde{B} d \dot{r}(t)+\widetilde{K} d r(t)
$$

where $\widetilde{B}$ and $\widetilde{K}$ are the coefficients of viscosity and stiffness, respectively, $\mathrm{Pb}(\mathrm{t})$ is the arterial pressure, and $\mathrm{r}(\mathrm{t})$ is the radius of the artery at the measurement part as calculated from the plethysmogram. In addition, to estimate the transfer function assuming the input parameter as the arterial pressure and the output parameter as the radius of the artery, Equation (1) can be described using the Laplace transform as follows:

$$
G_{p}(s)=\frac{C}{1+\tau S}
$$

where $C=1 / \widetilde{K}$ and $\tau=\widetilde{B} / \widetilde{K}$. In this study, we describe changes in arterial mechanical characteristics in response to mechanical stimuli using the stiffness value $\widetilde{K}$, which is the inverse of gain $C$ in compliance with the vessel and the time constant $\tau$

Usually, because blood vessel pressure and plethysmograms have individual differences, absolute values of viscoelasticity vary among individuals. Accordingly, we evaluated the regulation of biosignals between subjects by performing normalization with a value at the time of rest. The standard values $\overline{\widetilde{K}}$ and $\bar{\tau}$ are defined as the mean of a continuous 30-second period including the moment at which the minimum value was measured under the condition that the determination coefficient between estimation and measurement of blood pressure is greater than 0.9 . The normalized arterial wall viscoelasticity index values $\widetilde{K}_{n}$ and $\tau_{n}$ are defined as indexes for evaluation using $\overline{\widetilde{K}}$ and $\bar{\tau}$ as follows:

$$
\widetilde{K}_{n}=\frac{\widetilde{K}}{\widetilde{\widetilde{K}}_{r}}, \quad \tau_{n}=\frac{\tau}{\bar{\tau}_{r}}
$$

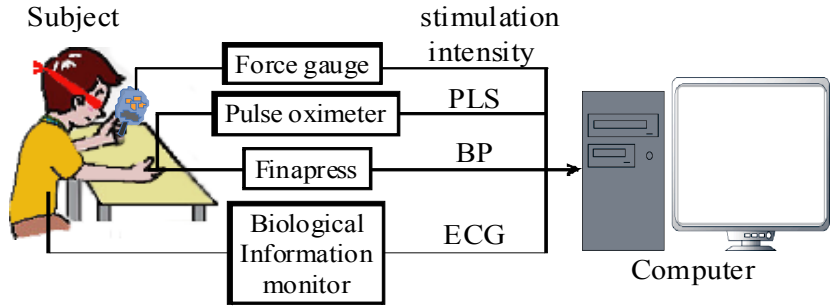

Figure 2. Experimental setup

To estimate the arterial wall viscoelasticity index for every heartbeat, the interval between each one is picked up from the electrocardiogram. In this method, the heartbeat interval is estimated using R-waves from among the QRS-waves measured when action potential spreads to the epicardium side from the endocardium side of the cardiac ventricle. When an $\mathrm{R}$-wave is measured, the viscoelasticity index is estimated using the previous $\mathrm{R}$-wave to ascertain the detected $\mathrm{R}$-wave interval, and real-time evaluation of the viscoelasticity index is enabled. Based on Section II. $A$, the viscoelasticity characteristics $\widetilde{K}$ and $\tau$ are estimated from blood pressure and the plethysmogram using the least-squares method, and $\widetilde{K}_{n}$ and $\tau_{n}$ are calculated using equation (3).

\section{B. Evaluation of Arterial Wall Viscoelasticity Index in Response to Mechanical Stimuli}

To verify the effectiveness of the proposed method, we conducted experiments to estimate the arterial wall viscoelasticity index in response to painful stimuli. Figure 2 illustrates the experimental setup. In total, eight 20-year-old male subjects were recruited. Each biosignal was measured from the right second finger, the right third finger and the chest, using a pulse oximeter (OLV-3100, Nihon Kohden), a noninvasive continuous sphygmomanometer (Finapres 2300, Ohmeda) and a cardiography device (BP-608EV, Colin Medical Technology), respectively. In the experiment, right hand fitted with the sensors, and part of the stimulator and the stimulated position at which the cone-shaped attachment (top corner $70^{\circ}$, Nidec-Shimpo) of the force gauge was pushed into the middle of the left palm. Using a holding fixture (FGS-50H, Nidec-Shimpo) for the stimulating device, the stimulus intensity was maintained at a constant level, and the stimulator was brought down from directly above onto the palm. All signals were simultaneously measured at $125 \mathrm{~Hz}$.

We evaluated viscoelastic characteristics in response to stimuli over a period of 60 seconds during which the subject's left hand was stimulated with a constant force. The stimulation intensities were set as $0[\mathrm{~N}], 1[\mathrm{~N}], 2[\mathrm{~N}]$ and $3[\mathrm{~N}]$. During the experiment, all subjects were sitting down in a resting state and could not see the stimulation part so as to avoid psychological influence from the sight of the stimulator. The measurements were repeated once for each stimulation intensity, and were performed in a resting state $(0-120$ [sec] $)$, a stimulated state $(120-180[\mathrm{sec}])$ and a resting state again $(180-240$ [sec] $)$. The arterial wall viscoelastic characteristics were estimated 


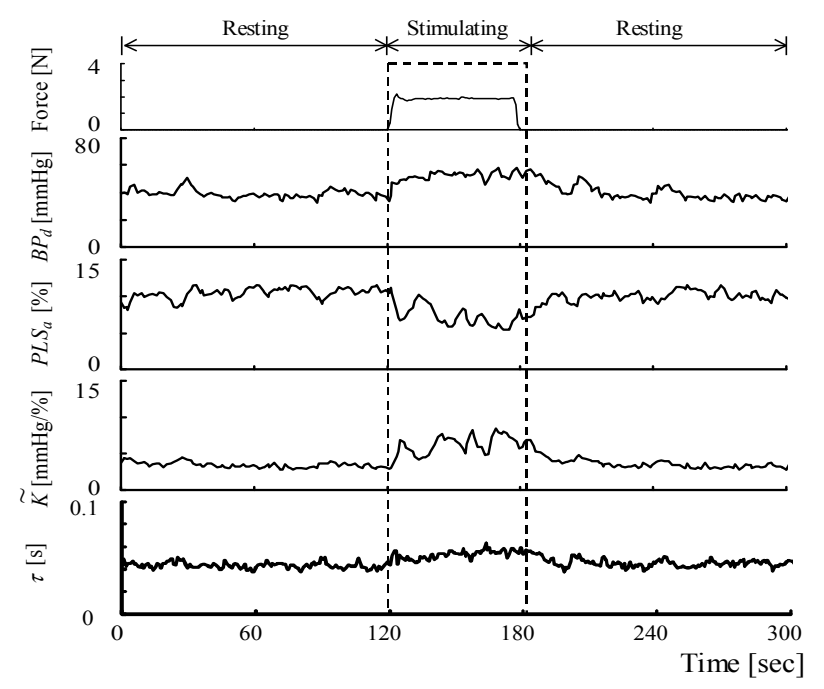

Figure 3. Estimated results of arterial wall impedance (Sub. A)

using the offline analysis method described in Section II. $A$. We compared the average for a 30 -second period in a stimulated state $(135-165$ [sec] $)$ with the each stimulation intensity.

All subjects were evaluated using VAS after the stimulation experiment, and stimuli were applied at each stimulation intensity level $(0[\mathrm{~N}], 1[\mathrm{~N}], 2[\mathrm{~N}], 3[\mathrm{~N}])$ for 60 seconds. VAS was explained to all subjects that the range of the scale ran from "No pain" to "Worst pain ever." All subjects marked the point corresponding to their level of pain intensity for each of the stimuli. All subjects were assessed four times with painful stimuli for each stimulation intensity, and therefore indicated four VAS values. The VAS value is the length from the left extremity to the point marked by the subject. We compared the valued of VAS and the average of $\widetilde{k}_{n}$ from a 30 -second period in a stimulated state $(135-165$ [sec]) with the reaction for each stimulation intensity, and used one way ANOVA for multiple comparisons. The level of significance was set to $P<0.05$.

\section{RESULTS}

Figure 3 shows the experimental results for Subject A, representing the results of $2[\mathrm{~N}]$ stimulation, and plots the stimulation intensity force, the pulse pressure (the difference between the highest and lowest blood pressure measurements) $B P_{\mathrm{d}}$, the plethysmogram amplitude $P L S_{\mathrm{a}}$, the value of stiffness $\widetilde{K}$ and the time constant $\tau$. The shaded areas surrounded with a dashed line correspond to the stimulation period. The results show small changes in the pulse pressure and plethysmogram as a result of stimulation, and indicate that the value of stiffness $(\widetilde{K})$ increased drastically and rapidly in the stimulation period $(120-180$ [sec] $)$. However, there was little change in the time constant $(\tau)$.

Figure 4 shows the average biological signals and estimated parameters in a stimulated state for each stimulation intensity. In the outcome for $B P_{d}$, there are no significant



Figure 4. Mean values of normalized signals during stimulation

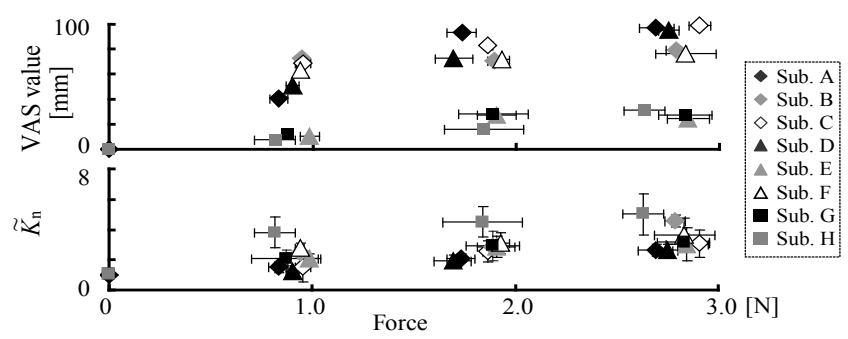

Figure 5. Comparison between VAS and stiffness values $\left(\widetilde{K}_{n}\right)$

changes with a stimulus intensity of $1-2[\mathrm{~N}]$ or $2-3[\mathrm{~N}]$ for some subjects. On the other hand, in the results of $P L S_{a}$ and $\widetilde{K}$, there are significant differences for almost all subjects. However, the results for $\tau$ generally show little significant difference, and demonstrate no relationship with stimulation intensity. In contrast, $P L S_{a}$ indicated significant differences ( $p$ $=0.0001)$ for a stimulus intensity of $0-1[\mathrm{~N}]$ for all subjects. With other stimuli of $1-2[\mathrm{~N}]$ and $2-3[\mathrm{~N}]$, there were significant differences ( $p=0.037$ and $p=0.034$, respectively) with eight subjects. The comparison of $\widetilde{K}$ values indicated significant differences $(p=0.007)$ for a stimulus intensity of 0 $-1[\mathrm{~N}]$ for all subjects. Additionally, with other stimuli of $1-2$ $[\mathrm{N}]$ and $2-3[\mathrm{~N}]$, there were significant differences $(p=0.014$ and $p=0.018$, respectively) with eight subjects.

Figure 5 shows a comparison between the conventional method VAS and the proposed stiffness values $\left(\widetilde{K}_{n}\right)$. The value of $\widetilde{K}_{n}$ is the average value of stiffness $\left(\widetilde{K}_{n}\right)$ during the stimulation period $(135-165[\mathrm{~N}])$. The VAS value and the stiffness value tended to increase with higher stimulation intensity in all subjects. We also compared the coefficients of 
variance variability during each level of stimulation intensity for all subjects. The coefficients of variance for VAS were $0.67,0.51$ and 0.50 for $1[\mathrm{~N}], 2[\mathrm{~N}]$ and $3[\mathrm{~N}]$, respectively, while the values for $\widetilde{K}_{n}$ were $0.37,0.27$ and 0.26 for $1[\mathrm{~N}], 2$ $[\mathrm{N}]$ and $3[\mathrm{~N}]$, respectively. In addition, VAS has limitations in terms of measurement on the right-side end of the scale at the maximum point. Here, the results of subjects A, C and D show that they assessed the level of pain at this extremity for a stimulation intensity of $3[\mathrm{~N}]$, meaning that VAS could not be used to evaluate pain for a mechanical stimulation level of 3 $[\mathrm{N}]$ or greater.

\section{DISCUSSION}

It was possible to ascertain changes in viscoelasticity in response to stimulation using the proposed system. In this study, the stiffness changes for each level of stimulation intensity are compared, and the accuracy of the stiffness measurements and VAS is verified. The usability of stiffness as a metric is also discussed. Figure 4 shows the average of each biological signal and the estimated parameters $\left(B P_{d}, P L S_{a}\right.$, $\widetilde{K}_{n}$ and $\tau_{n}$ ) in the stimulation state for each stimulation intensity $(0,1,2$ and $3[\mathrm{~N}]$, respectively). These parameters' values were normalized using the value of the resting state. The results show a poor correlation between stimulation intensity and $B P_{d}$ for most subjects. In the results for Subject $\mathrm{A}$, for example, $B P_{d}$ increased with higher stimulation at $2[\mathrm{~N}]$, but $B P_{d}$ decreased for a stimulation level of $3[\mathrm{~N}]$ in comparison to that of $2[\mathrm{~N}]$. On the other hand, the results for $P L S_{a}$ and $\widetilde{K}_{n}$ indicated significant differences $(\mathrm{P}<0.01)$ for different levels of stimulation intensity. $P L S_{a}$ tended to decrease with increased stimulation, and $\widetilde{K}_{n}$ tended to increase. Both tendencies (i.e., a decrease in $P L S_{a}$ and an increase in $\widetilde{K}_{n}$ ) were confirmed with all subjects.

This section considers the cause of the VAS value reaching the assessable limit for a stimulation intensity of $3[\mathrm{~N}]$ as seen in Fig. 5. As VAS evaluation depends on the experience of the subject, the results may vary between subjects for the same level of stimulation intensity, and the maximum limit of assessment may be different. On the other hand, stiffness shows a similar tendency among subjects in response to increasing stimulation intensity, meaning that comparison and evaluation between subjects may be possible. The variation coefficient of $\widetilde{K}_{n}$ for each stimulation intensity was relatively small in comparison to the values of VAS. In addition, because $\widetilde{K}_{n}$ tended to increase in a linear fashion for a stimulation intensity of $3[\mathrm{~N}]$, evaluation in other stimulation intensity experiments can be expected. The changes in stiffness were caused by a mixed reaction of spinal reflex and autonomic nerve activity in response to pain and stimulation. The evaluation of arterial stiffness is equivalent to evaluating this mixed reaction, and has the potential to allow objective evaluation of the subject's degree of pain. Conversely, as previously indicated, VAS evaluation is influenced by subjectivity.

\section{CONCLUSIONS}

In this study, we proposed a new arterial wall viscoelasticity-based evaluation method that allows assessment of biological responsiveness to mechanical painful stimuli. Its effectiveness was confirmed through the experiments that involved evaluating changes in arterial wall viscoelasticity for different levels of stimulation intensity and comparing the results with the conventional VAS method. The results showed that arterial viscoelasticity has the potential to allow objective evaluation for pain. However, as the effects of pain are not limited to changes in arterial wall viscoelasticity, it is also necessary to consider influences in other areas. In the future, research will be conducted to consider a method for more accurate pain evaluation by examining the influence of other factors and to evaluate small differences between individuals in stiffness value that are considered to have caused organic changes in the arterial wall.

\section{ACKNOWLEDGMENT}

The authors would like to thank all participants in this study and cordially grateful to Mr. Akinobo Kouno of the TOYOBO Co., Ltd for manpower to conduct the experiment.

\section{REFERENCES}

[1] K. Toyota, S. Sakura, "Choice of Concentration, Volume and Dosage of Local Anesthetics in Epidural Anesthesia," The Journal of Japan Society for Clinical Anesthesia, vol. 28, 79-84, 2008.

[2] K. Ho, J. Spence, MF. Murphy, "Review of pain-measurement tools," Ann EmergMed, 27, pp. 427-432, 1996.

[3] EC. Huskinsson, "Measurement of pain, " Lancet, vol. 2, pp. 1127-1131, 1974.

[4] L. Thomas, L. Irene, D. Lisbeth et al., "Reliability and Responsiveness of Three Different Pain Assessments," J Rehabil Med, vol.33, pp. 279283, 2001.

[5] J. Colleen, Dunwoody, AK. Dina, P. Chris et al., "Assessment, Physiological Monitoring, and Consequences of Inadequately Treated Acute Pain," Journal of Peri-Anesthesia Nursing, vol.23(1), pp. 15-27, 2007.

[6] H. Shimazu, S. Seno, S. Kato, H. Kobayashi, M. Akimoto, "Development of a Quantitative Measurement Method for the Magnitude of Pain Using Painless Electrical Stimulation and Its Evaluation Using Experimental Pain," Japanese Sociey for Medical and Biological Engineering, vol.43(1), pp. 117-123, 2005.

[7] C. Rene, "Pain:Mechanisms and Management," Ishiyaku Publishers, Inc., Tokyo, pp. 33-49, 1994.

[8] H. Kawamura, M. Tsujishita, D. Okazaki et al., "Pain Evaluation using Pain Related Magnetic Encephalography," The Journal of Japanese Physical Therapy Association, vol.31(2), pp. 177, 2004.

[9] M. Akamatsu "Pain and Evaluation," Journal of the Society of Biomechanisms, vol.14(3), pp. 151-159, 1990.

[10] J. Hasegawa, D. Suguyama, M. Kumasaka et al., "Evaluation of pain treatment by PainVision," The journal of the Japan Society of Pain Clinicians, vol.15(2), pp. 144-149, 2008.

[11] A. Kutluk, T. Tsuji, T. Ukawa, R. Nakamura, N. Saeki, M. Yoshizumi, M. Kawamoto, "A Novel Online Method to Monitor Autonomic Nervous Activity Based on Arterial Wall Impedance and Heart Rate Variability," Medical and Biological Engineering and Computing, vol.48(4), pp. 351-359, 2010.

[12] A. Sakane, T. Tsuji, Y. Tanaka, N. Saeki, and M. Kawamoto, "Monitoring of Vascular Conditions Using Plethysmogram," Transactions of the Society of Instrument and Control Engineers, 40(12), pp. 1236-1242, 2004. 